\title{
Partial splenectomy for massive splenomegaly secondary to Gaucher's disease
}

\author{
W.E.G. Thomas and D.A. Winfield \\ Departments of Surgery and Haematology, Royal Hallamshire Hospital, Glossop Road, Sheffield S10 2JF, UK
}

Summary: A 16 year old girl with Type 1 Gaucher's disease presented with massive splenomegaly, hypersplenism and abdominal discomfort. Traditionally hypersplenism has been treated with splenectomy, but this results in a high incidence of overwhelming sepsis and accelerated sphingolipid deposition in both liver and bone. A $90 \%$ partial splenectomy was therefore performed leaving a fully vascularized inferior segment of the spleen and resecting $5.8 \mathrm{~kg}$ of splenic tissue. The patient made an uneventful recovery with a marked improvement in her haematological parameters and general condition.

\section{Introduction}

Gaucher's disease is an autosomal recessive genetic disorder resulting in a deficiency of B-glucocerebrosidase $^{1}$ which leads to an accumulation of glucocerebroside in the reticuloendothelial system and bone marrow. The principal clinical presentations of patients with Gaucher's disease are hepatosplenomegaly and bone pain. Massive splenomegaly with hypersplenism is therefore a common presentation in Type 1 Gaucher's disease (adult form) and in the past this has been treated by splenectomy. ${ }^{2,3}$ However, progressive hepatomegaly and accelerated bone disease have been reported after this procedure, ${ }^{2,3}$ as have overwhelming septic complications that can be fatal. ${ }^{4}$ These results have suggested that patients with massive splenomegaly secondary to Gaucher's disease may benefit from a partial splenectomy. A partial splenectomy procedure was first described in $1869,{ }^{5}$ and subsequently has been utilized following trauma ${ }^{6-8}$ for splenic cysts ${ }^{9,10}$ and haematological disorders. ${ }^{11-13}$ Partial splenectomy for Gaucher's disease has been reported in Israel ${ }^{14,15}$ and the USA, ${ }^{16,17}$ but we now report the first documented case in the British literature in one of the largest spleens in which this procedure has been attempted.

\section{Patient and methods}

A 16 year old female weighing $35 \mathrm{~kg}$ presented with progressive symptoms of lethargy, abdominal pain

Correspondence: W.E.G. Thomas, F.R.C.S., M.S.

Accepted: 21 May 1991 and amenorrhoea and was found to have massive splenomegaly to the level of her pelvis (Figure 1). Four years previously she had been diagnosed as having aseptic femoral head necrosis. Peripheral blood results were haemoglobin $7.3 \mathrm{~g} / \mathrm{dl}$, white count $2.7 \times 10^{9} / 1$ and platelets $44 \times 10^{9} / 1$. Bong marrow trephine biopsy showed extensive infiltras tion by Gaucher's cells and the diagnosis of Type $\bar{\Phi}$ Gaucher's disease was confirmed by reduceฮ ' plasma B-glucosidase activity and increased levels of acid phosphatase and angiotensin converting enzyme. X-rays of her skeleton showed signs of old avascular necrosis of the right femoral head (Figure 2) and abnormal trabecular pattern of both proximal femora.

One possible treatment option was allogeneic bone marrow transplantation but this could not be undertaken since her unaffected sister was HLA incompatible. Because of symptoms associated with her large spleen, it was decided to proceed to partial splenectomy to try and avoid rapid deterioration of her skeletal problems.

Pre-operatively the patient received pneumococcal vaccine, a transfusion of platelets and cefuroxime prophylaxis. Laparotomy revealed massive splenomegaly, no splenunculus and moderate hepatomegaly. The initial operative manoeuvre was to open the lesser sac and place a rubber sling around the splenic artery as it ran along the upper surface of the pancreas. The spleen was then mobilized dividing the spleno-renal ligament and omental and diaphragmatic attachments. The short gastric vessels were divided and the spleen delivered into the wound. Hilar dissection then started at the upper pole, ligating and dividing all vessels except for the most inferior artery and 


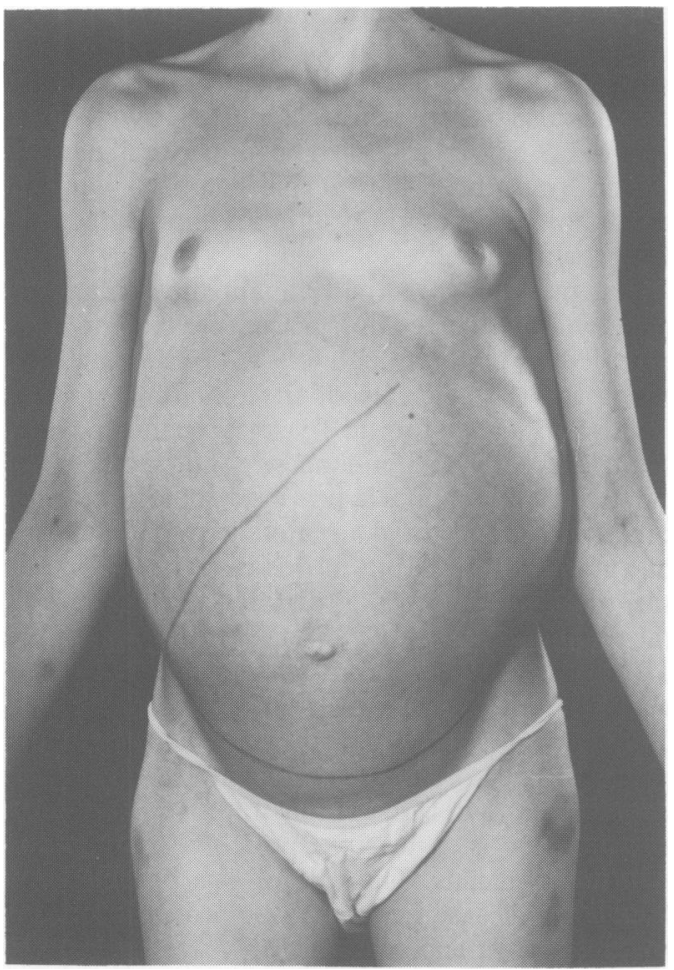

Figure 1 Massive splenomegaly.

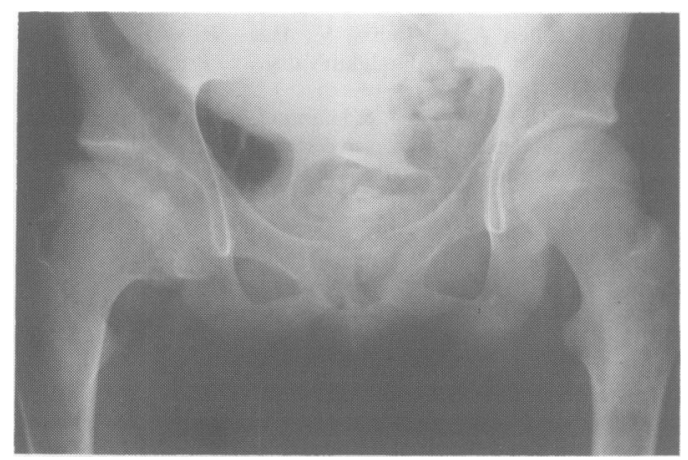

Figure 2 Right sided aseptic femoral head necrosis secondary to Gaucher's disease.

vein supplying the lower pole of the spleen (Figure 3). The devascularized portion of the spleen became less tense and a clear line of demarcation was seen. The spleen was divided along the demarcation line and a $90 \%$ splenectomy performed resecting $5.8 \mathrm{~kg}$ of spleen $(14 \%$ of body weight: normal splenic/body weight ratio $0.3 \%$ ). Pressure was applied to the cut surface and the spleen was

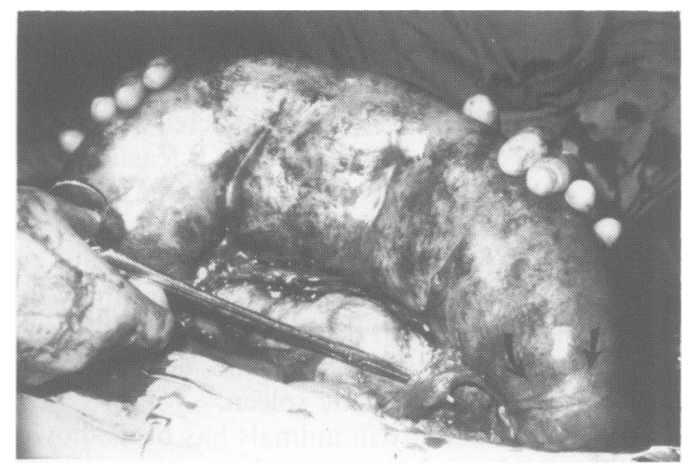

Figure 3 The spared inferior artery and vein to the lower pole of the spleen being elevated by the artery clip, and the line of demarcation (arrowed).

wrapped in a mesh of absorbable polyglycolic acid with a layer of the haemostatic substance spongistan over the cut surface. The spleen was then sutured to the diaphragm in 3 sites using the mesh to prevent torsion.

The patient made an uneventful recovery with minimal drainage. She was discharged on the tenth day following surgery.

At follow-up the patient has made excellent progress. Her haemoglobin, platelets and white cell count 6 months later were $12.9 \mathrm{~g} / \mathrm{dl}, 130 \times 10^{9} / 1$ and $8.2 \times 10^{9} / 1$ respectively and her weight $50 \mathrm{~kg}$. Her periods are regular and normal. Clinical examination and CT scan give no indication of progressive enlargement of the remnant at 7 months and an isotope scan shows normal splenic perfusion.

\section{Discussion}

The management of patients with massive splenomegaly and hypersplenism secondary to Type I Gaucher's disease has been unsatisfactory. In the past the only option was to perform a splenectomy, but this was often accompanied by a high incidence of sepsis and even death. ${ }^{4}$ The immune system is further embarrassed in these patients by the infiltration of the remaining reticuloendothelial system by the deposition of the Gaucher cells, placing these patients at even greater risk of sepsis following splenectomy than those undergoing such surgery for trauma, immune thrombocytopenic purpura or hereditary spherocytosis. Furthermore, after splenectomy in patients with Gaucher's disease, there is often a progression in the hepatomegaly and in bone disease; in this patient, who was already having trouble with her right hip due to aseptic femoral 
head necrosis, this would have been disastrous. Other treatment options were therefore considered.

Allogeneic bone marrow transplantation could not be undertaken since her unaffected sister was HLA incompatible. However, even if feasible, this would not have addressed the issue of her abdominal pain and difficulties in moving due to the splenic bulk. Arterial embolization can be considered $^{18}$ but has to be done in stages, may be accompanied by a high fever and marked discomfort due to splenic infarction and does nothing initially for the bulk of the spleen. ${ }^{17}$

Partial splenectomy in animals has been shown to inflict far less of an onslaught on the immune system than total splenectomy, ${ }^{19}$ and in Gaucher's disease would decrease the bulk without increasing significantly the hepatomegaly or bone disease.

The actual procedure of partial splenectomy for such a vast spleen is a daunting prospect. However, with meticulous surgical technique, it is a feasible and safe procedure. In this patient no postoperative complications occurred, but in the cases reported from Israel and the USA a few complications have been reported including torsion of the splenic remnant, ${ }^{14}$ haemorrhage,${ }^{14}$ necrosis of the remnant ${ }^{15}$ and left-sided pleural effusion. ${ }^{17}$

Partial splenectomy in these patients undoubtedly improves the state of hypersplenism, and is also accompanied by an increase in body weight, muscle

\section{References}

1. Brady, R.O., Kanfer, J.N. \& Shapiro, D. Metabolism of glucocerebrosides. Evidence of an enzymatic deficiency in Gaucher's disease. Biochem Biophys Res Commun 1965, 18: 221-225.

2. Salky, B., Kreel, I., Gelernt, I. et al. Splenectomy for Gaucher's disease. Ann Surg 1979, 190: 592-594.

3. Shiloni, E., Bitran, D., Rachmilewitz, E. et al. The role of splenectomy in Gaucher's disease. Arch Surg 1983, 118: 929-932.

4. Walker, W. Splenectomy in childhood: a review in England and Wales 1960-1964. Br J Surg 1976, 63: 36-43.

5. Pean, J. Ovariotomie et splenotomie, 2nd ed. Germer-Bailliere, Paris, 1869, pp. 129-138.

6. Buntain, W.L. \& Lynn, H.B. Splenectomy: changing concepts for the traumatised spleen. Surgery 1979, 86: 748-759.

7. Morgenstern, L. \& Shapiro, S.J. Techniques of splenic conservation. Arch Surg 1979, 114: 449-454.

8. Sherman, R. Perspective in management of trauma to the spleen. J Trauma 1980, 20: 1-13.

9. Brown, J.J., Bynde, R.P., Greene, F.L. et al. Splenic salvage techniques in the management of pseudocysts of the spleen. South Med J 1986, 79: 710-711.

10. Morgenstern, L. \& Shapiro, S.J. Partial splenectomy for non-parasitic splenic cysts. Am J Surg 1980, 139: 278-281.

11. Kheradpir, M.H. \& Alebouyeh, M. Partial splenectomy in the treatment of thalassaemia major. $Z$ Kinderchir 1985, 40: $195-198$. bulk and tone of abdominal musculature. ${ }^{15}$ It is of interest to note that our patient commenced her menses within 2 months of surgery and one similar case has also been previously reported in which a 20 year old female commenced having normal periods within 2 months of partial splenectomy, ${ }^{17}$ while in children it appears to result in an improvement in the growth curve. ${ }^{15}$

The rate at which the remnant may enlarge is unknown and as yet this procedure has not been attended by a long enough follow-up adequately to assess the ultimate fate of the remaining spleen. Follow-up scans of up to 1 year have shown some slight increase in remnant size but nothing of clinical significance. ${ }^{16}$ It has been the objective of other workers to leave a remnant roughly the size of a normal spleen, but in this case the enormous size of the spleen $(5.8 \mathrm{~kg})$ dictated that the size of the remnant depended on the vascular supply. Thus the remnant was that part of the spleen that was adequately vascularized on only a single vessel. To have removed more would have run this risk of potential haemorrhagic complications.

This case therefore demonstrates that a partial splenectomy is a feasible possibility even with enormous spleens. The role of this procedure in Gaucher's disease remains to be proven, but thus far looks most encouraging.

12. Holschneider, A.M., Lohrs, V., Haas, R. et al. Selective hemisplenectomy for Hodgkin's disease. Prog Pediatr Surg 1985, 18: 162-168.

13. Boles, E.T., Haase, G.M. \& Hamoudi, A.B. Partial splenectomy in staging laparotomy for Hodgkin's disease: an alternative approach. J Paediat Surg 1979, 13: 581-586.

14. Bar-Maor, J.A. \& Govrin-Yedudain, J. Partial splenectomy in children with Gaucher's disease. Pediatrics 1985, 76: 398-401.

15. Rubin, M., Yampolski, I., Lambrozo, E. et al. Partial splenectomy in Gaucher's disease. J Paediat Surg 1986, 21: 125-128.

16. Rodgers, B.M., Tribble, C. \& Joob, A. Partial splenectomy for Gaucher's disease. Ann Surg 1987, $205:$ 693-699.

17. Guzzetta, P.C., Connors, R.H., Fink, J. et al. Operative techniques and results of subtotal splenectomy for Gaucher's disease. Surg Gynec Obstet 1987, 164: 359-362.

18. Spigos, D.G., Jonasson, O., Mozes, M. et al. Partial splenic embolisation in the treatment of hypersplenism. Am J Radiol 1979, 132: 777-782.

19. Cooney, D.R., Dearth, J.C., Swanson, S.E. et al. Relative merits of partial splenectomy, splenic reimplantation and immunisation in preventing post-splenectomy infection. Surgery 1979, 86: 561-569. 